\title{
Distortion of the Benzene Ring in 1,2,3-Trifluorobenzene; A High-Resolution Molecular Beam Fourier Transform Microwave Study
}

\author{
Ulrich Wolschendorf, Uwe Kretschmer, and Dieter H. Sutter \\ Institut für Physikalische Chemie, Christian-Albrechts-Universität, \\ Olshausenstr. 40 - 60, D-24098 Kiel \\ Z. Naturforsch. 51 a, 46-52 (1996); received November 10, 1995
}

\begin{abstract}
The results of a molecular beam microwave Fourier transform study of 1,2,3-trifluorobenzene and its monosubstituted ${ }^{13} \mathrm{C}$-isotopomers, all observed in natural abundance, are presented. Typically 30 transitions were observed for each isotopomer. Centrifugal perturbation theory up to fourth order was used to analyse the observed rotational spectra. Due to the high sensitivity and resolution of the spectrometer, very accurate vibronic ground state rotational constants can be reported:

$\begin{array}{cccccc} & { }^{13} \mathrm{C}_{2} & { }^{13} \mathrm{C}_{3} & { }^{13} \mathrm{C}_{4} & { }^{13} \mathrm{C}_{5} & { }^{12} \mathrm{C} \\ \mathrm{A} / \mathrm{MHz} & 2315.26797(10) & 2319.63903(10) & 2298.06711(11) & 2272.17274(13) & 2319.60029(06) \\ B / \mathrm{MHz} & 1757.93383(14) & 1749.22591(17) & 1748.64646(17) & 1757.84791(25) & 1757.83010(08) \\ \mathrm{C} / \mathrm{MHz} & 999.16620(09) & 997.15481(08) & 992.96022(09) & 991.02463(07) & 999.93756(10)\end{array}$
\end{abstract}

They are used to derive a partial $r_{0}$-structure, which shows the distortions of the benzene ring caused by fluorine substitution.

\section{Introduction}

We have initiated this study in the context of our continuing interest in the rotational Zeeman effect of substituted aromatic six-membered rings [1, 2, 3, 4]. Accurate structural information is a prerequisite, if one wants to further evaluate the information contained in the measured molecular $g$ - and $\xi$-tensor elements [5].

The rotational spectrum of the most abundant isotopomer of 1,2,3-trifluorobenzene has been carefully reassigned just recently [6]. In the following we report our results for the four monosubstituted ${ }^{13} \mathrm{C}$ isotopomers. All spectra were observed in natural abundance by pulsed nozzle molecular beam Fourier transform microwave spectroscopy (MB-FTMW).

Combined with the results for the most abundant isotopomer, a set of effectively ten rotational constants is now available for this presumably planar molecule. If a planar equilibrium geometry with $\mathrm{C}_{2 \mathrm{v}}$ symmetry is assumed, eleven internal coordinates (bond angles and bond distances) are sufficient for a complete description of the molecular structure. In other words, the present experimental data should

Reprint requests to Prof. D. H. Sutter;

Fax: (0431) 880-1416. allow for an almost complete $r_{0}$-structure [7] determination. Correlations however reduce the number of determinable parameters to seven (see below).

\section{Experimental Details}

The sample of 1,2,3-trifluorobenzene (98\% purity) was purchased from Lancaster Synthesis $\mathrm{GmbH}$, Mülheim am Main. It was used after several bulb to bulb distillations without further purification. To observe the spectrum we used our MB-MWFT spectrometer, originally designed for trace gas detection [8]. The spectrometer was improved to include automatic scanning capabilities. The present setup is given in detail in $[9,10]$. In Fig. 1 we present a $135 \mathrm{MHz}$ section out of a larger scan.

Trifluorobenzene was deluted (1\%) in argon as carrier gas. The backing pressure was kept below 50 $\mathrm{kPa}(0.5 \mathrm{~atm})$. Such a comparatively low backing pressure reduces the probability of van der Waals complex formation. The parallel beam arrangement (compare Fig. 2) was used. Due to the large interaction zone in the cavity resonator, this arrangement, which was first introduced by Grabow [11, 12], gives considerably higher sensitivity and resolution than the original Flygare-Balle perpendicular beam arrangement [13]. The spectrometer not only is extremely sensitive but, since high- $J$ transitions can be virtually suppressed, 


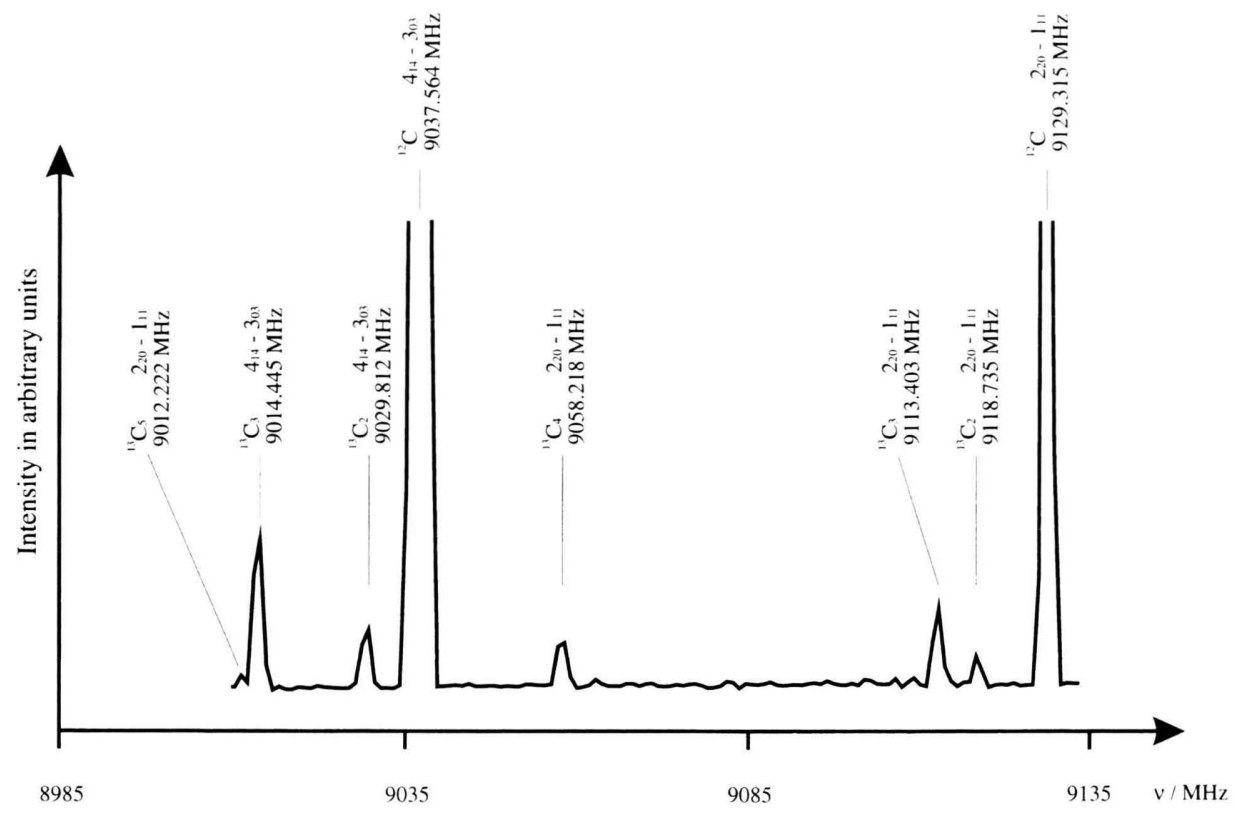

Fig. 1. Scan of the frequency region from 9000 to $9135 \mathrm{MHz}$. Experimental conditions: polarization frequency stepwidth: $910 \mathrm{kHz}$, at each polarization frequency 128 transient emission signals are averaged up for noise reduction, for each transient emission signal $2 \mathrm{k}$ data points are taken at a sample interval of $40 \mathrm{~ns}$, polarization pulsewidth: $0.5 \mu \mathrm{s}$, polarization power: $4 \mathrm{~mW}$, time needed for the scan: $60 \mathrm{~min}$.

it is also ideally suited for the analysis and for the assignment of otherwise dense rotational spectra. The reason for the lack of high- $J$ transitions is twofold. First, due to the very effective rotational cooling in the expansion zone immediately downstream of the nozzle, only low- $J$ rotational states are populated at all. Second, since the different $M$-states within a higher- $J$ rotational transition are polarized at different speeds according to their different dipole matrix elements, $M$-dephasing during excitation leads to an additional drop in the intensity of high- $J$ free induction decay signals. With increasing $J$-values the latter effect becomes more and more important, since more $M$-substates contribute to the corresponding emission signal. To summarize: by the proper choice of the backing pressure ( $\rightarrow$ cooling) and the duration of the polarizing pulse ( $\rightarrow$ dephasing) the spectrometer can be set to conditions under which only the lowest rotational transitions are observed. This considerably facilitates the assignment.

\section{Assignment of the ${ }^{13} \mathrm{C}$-Spectra}

Based on the results of an earlier investigation of 1,2-difluorobenzene, 1,2,4-trifluorobenzene, and 1,2,3,5-tetrafluorobenzene $[14,15]$, we were able to predict the low- $J$ transition frequencies of the four mono- ${ }^{13} \mathrm{C}$-substituted 1,2,3-trifluorobenzenes within better than $1 \mathrm{MHz}$. All could be observed with sufficient signal to noise ratios. As an example we present a high resolution mode registration of the $J_{K_{a}^{\prime} K_{c}^{\prime}}^{\prime}-J_{K_{a}^{\prime \prime} K_{c}^{\prime \prime}}^{\prime \prime}=2_{20}-1_{11}$ rotational transition of 1,2,3-trifluorobenzene- ${ }^{13} \mathrm{C}_{3}$ in Figure 3. This transition is also included in the broad band scan presented as Figure 1. Spin-rotation coupling splitting caused by the $\mathrm{F}$ - and $\mathrm{H}$-nuclei is still below the resolution of the instrument. The observed doublet splitting is caused by the Doppler-effect. (In the parallel beam arrangement the front mirror of the cavity detects a blue-shifted, the rear mirror a red-shifted molecular emission signal and the splitting carries information on the beam velocity. In our case, with argon as carrier gas, the beam velocity was around $560 \mathrm{~m} / \mathrm{s}$.)

In Table 1 we list part of the observed transition frequencies. The complete frequency listings are available from the authors. Typically they include about 30 transition frequencies for each isotopomer but for the remaining transitions the rotational quantum numbers are not always the same for all isotopic species. 


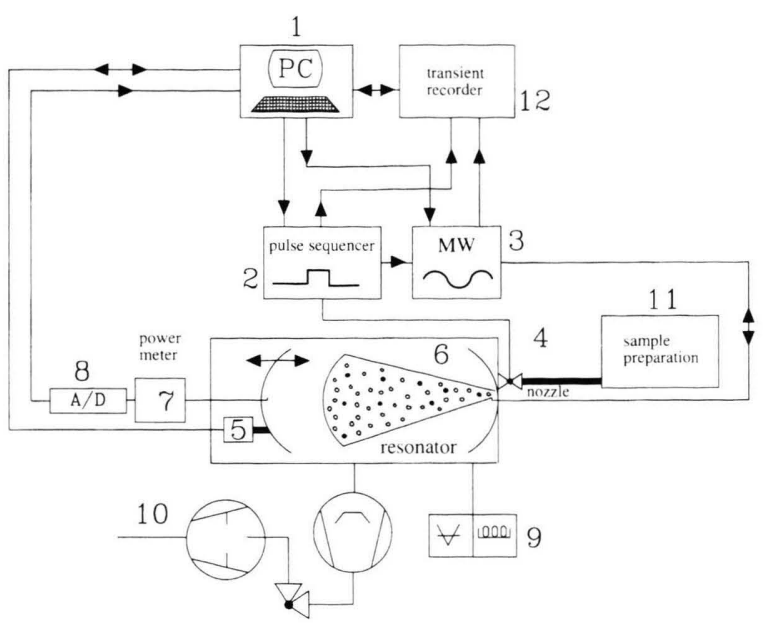

Fig. 2. General setup of the molecular beam FTMW spectrometer. 1. PC (80386 processor), 2. pulse sequencer (homemade), 3. microwave synthesizer (Systron-Donner 1730B, 2-26.5 GHz), 4. nozzle (General Valve), 5. step motor drive for the movable mirror, 6. Fabry-Perot resonator, 7. power meter (Hewlett Packard 437B), 8. A/D converter for power control (Analog Devices, AD 571), 9. pressure control: baratron (MKS 390), multigascontroler (MKS 147C), 10. vacuum system: diffusion pump (Balzers, DIF 250, 3100 l/s), rotary pump (Balzers, DUO $060 \mathrm{~A}, 68$ $\left.\mathrm{m}^{3} / \mathrm{h}\right)$, 11. sample preparation and reservoir, 12. transient recorder (TR-AS 102, Dr. Strauss System Elektronik).

The analysis of the spectra was carried out using Typke's ZFAP4 program (version 4.7) [16]. This program is based on Watson's Hamiltonian [17] and accounts for fourth order centrifugal corrections to the rigid rotor frequencies. In the present case, Watson's asymmetric top reduction was used in the III $^{r}$ representation. The corresponding effective Hamiltonian is

$$
\begin{aligned}
\hat{H}= & A \cdot \hat{P}_{a}^{2}+B \cdot \hat{P}_{b}^{2}+C \cdot \hat{P}_{c}^{2} \\
& -\Delta_{J} \cdot \hat{P}^{4}-\Delta_{J K} \cdot \hat{P}^{2} \hat{P}_{c}^{2}-\Delta_{K} \cdot \hat{P}_{c}^{4} \\
& -\delta_{J} \cdot \hat{P}^{2}\left(\hat{P}_{a}^{2}-\hat{P}_{b}^{2}\right) \\
& -\delta_{K} \cdot\left[\hat{P}_{c}^{2}\left(\hat{P}_{a}^{2}-\hat{P}_{b}^{2}\right)+\left(\hat{P}_{a}^{2}-\hat{P}_{b}^{2}\right) \hat{P}_{c}^{2}\right] .
\end{aligned}
$$

As can be seen from Table 1, the agreement between experimental and calculated frequencies is better than $1 \mathrm{kHz}$ for the low- $J$-transitions observed here. This results in 7-digit accuracies for the rotational constants. They are presented in Table 2 together with the fourth order centrifugal distortion constants.

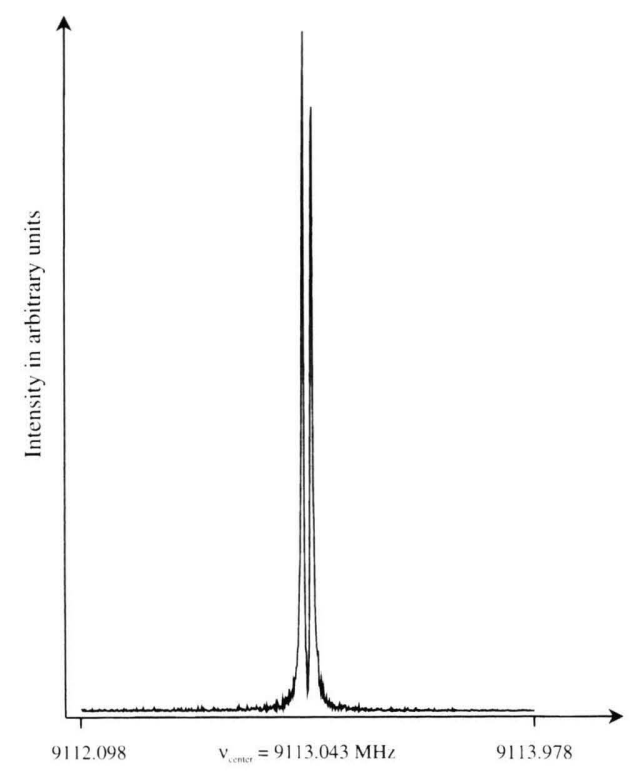

Fig. 3. $J_{K_{a}^{\prime} K_{c}^{\prime}}^{\prime}-J_{K_{a}^{\prime \prime} K_{c}^{\prime \prime}}^{\prime \prime}=2_{20}-1_{11}$ transition of the ${ }^{13} \mathrm{C}_{3}-$ isotopomer of 1,2,3-trifluorobenzene. Central frequency of the Doppler dublet: $9113.043 \mathrm{MHz}$. Doppler splitting: 38 $\mathrm{kHz}$. The given power spectrum was detected using $8 \mathrm{k}$ data points at a sample interval of $10 \mathrm{~ns}$, polarization frequency: 9113.05 MHz.

\section{Derivation of the Molecular Structure}

\section{a) Introductory Remarks}

The observed effective rotational constants carry information on the molecular geometry. To a high degree of approximation they correspond to vibrational expectation values of the principal moments of inertia [7]:

$$
\begin{aligned}
& A_{v_{1}, \ldots, v_{30}}^{(i)}= \\
& \frac{h}{8 \pi^{2}} \cdot\left\langle v_{1} \ldots\left|\frac{1}{\sum m_{n} \cdot\left(b_{i, n}^{2}+c_{i, n}^{2}\right)}\right| v_{1}, \ldots\right\rangle, \\
& B_{v_{1}, \ldots, v_{30}}^{(i)}= \\
& \frac{h}{8 \pi^{2}} \cdot\left\langle v_{1} \ldots\left|\frac{1}{\sum m_{n} \cdot\left(c_{i, n}^{2}+a_{i, n}^{2}\right)}\right| v_{1} \ldots\right\rangle, \\
& C_{v_{1}, \ldots, v_{30}}^{(i)}={ }^{\frac{h}{8 \pi^{2}}} \cdot\left\langle v_{1} \ldots\left|\frac{1}{\sum m_{n} \cdot\left(a_{i, n}^{2}+b_{i, n}^{2}\right)}\right| v_{1}, \ldots\right\rangle
\end{aligned}
$$

$\left(m_{n}=\right.$ atomic mass of the $n$-th atom; $a_{i, n} \cdot b_{i, n}, c_{i, n}$ its coordinates with respect to the principal inertia 
Table 1. Rotational transitions $J_{K_{a}^{\prime}}^{\prime} K_{c}^{\prime}-J_{K_{a}^{\prime \prime} K_{c}^{\prime \prime}}^{\prime \prime}$ and experimental frequencies $\nu_{\text {exp }} / \mathrm{MHz}$ of the ${ }^{13} \mathrm{C}$-isotopomers of $1,2,3$ trifluorobenzene. In brackets : $\nu_{\text {calc }}-\nu_{\text {exp }}$.

\begin{tabular}{cccccc}
\hline$J^{\prime}-J^{\prime \prime}$ & $\nu_{\exp }\left({ }^{13} \mathrm{C}_{2}\right)$ & $\nu_{\exp }\left({ }^{13} \mathrm{C}_{3}\right)$ & $\nu_{\exp }\left({ }^{13} \mathrm{C}_{4}\right)$ & $\nu_{\exp }\left({ }^{13} \mathrm{C}_{5}\right)$ & $\nu_{\exp }\left({ }^{12} \mathrm{C}\right)$ \\
\hline $2_{21}-1_{10}$ & $7944.9666(-6)$ & $7956.0679(1)$ & $7887.1582(-2)$ & $7807.5417(3)$ & $7958.7344(6)$ \\
$2_{20}-1_{11}$ & $9118.7347(3)$ & $9113.0426(4)$ & $9058.2177(3)$ & $9012.2218(2)$ & $9129.3151(-1)$ \\
$3_{31}-2_{20}$ & $12756.0688(2)$ & $12777.7456(4)$ & $12662.0300(0)$ & $12524.5313(-3)$ & $12779.2731(-1)$ \\
$3_{22}-2_{11}$ & $9943.2943(-3)$ & $9950.3735(-5)$ & $9873.0733(-3)$ & $9789.5852(-2)$ & $9958.6043(-3)$ \\
$3_{30}-2_{21}$ & $13340.2143(6)$ & $13344.2506(4)$ & $13247.8291(-1)$ & $13151.5197(3)$ & $13359.1681(9)$ \\
$4_{32}-3_{21}$ & $14853.4246(4)$ & $14874.9072(-2)$ & $14745.0984(6)$ & $14593.9770(0)$ & $14879.3810(10)$ \\
$4_{14}-3_{03}$ & $9029.8122(-2)$ & $9014.4452(-2)$ & $8972.8737(3)$ & $8949.2743(7)$ & $9037.5637(3)$ \\
$4_{41}-3_{30}$ & $17547.8185(5)$ & $17576.2668(2)$ & $17418.8781(-1)$ & $17232.7929(11)$ & $17579.3557(3)$ \\
$4_{23}-3_{12}$ & $11599.8069(1)$ & $11603.0041(9)$ & $11519.5057(3)$ & $11435.0351(-1)$ & $11616.2236(-6)$ \\
$4_{40}-3_{31}$ & $17773.7406(4)$ & $17790.9470(0)$ & $17646.9061(-1)$ & $17489.3732(8)$ & $17802.3493(-3)$ \\
$4_{04}-3_{13}$ & $8961.9785(5)$ & $8941.4313(-3)$ & $8907.0755(5)$ & $8895.1179(1)$ & $8968.2043(7)$ \\
$4_{31}-3_{22}$ & $17396.1288(2)$ & $17353.2644(-4)$ & $17290.8693(-3)$ & $17283.4614(-4)$ & $17407.2090(0)$ \\
$4_{13}-3_{22}$ & $10327.8976(4)$ & $10270.9853(7)$ & $10274.8969(1)$ & $10337.0646(4)$ & $10325.7779(1)$ \\
$5_{14}-5_{05}$ & $8811.1659(1)$ & $8781.7821(-1)$ & $8760.0136(4)$ & $8768.6904(-4)$ & $8814.7492(-2)$ \\
$5_{33}-4_{22}$ & $16461.1792(-2)$ & $16483.5636(4)$ & $16341.6044(-4)$ & $16178.3426(-6)$ & $16489.5262(-2)$ \\
$5_{24}-4_{13}$ & $13240.9620(0)$ & $13231.6948(2)$ & $13153.4037(3)$ & $13089.1751(9)$ & $13256.0292(-2)$ \\
$5_{41}-4_{32}$ & $21263.3013(-3)$ & $21237.8251(-1)$ & $21126.2731(9)$ & $21054.6618(2)$ & $21284.4021(9)$ \\
$5_{05}-4_{14}$ & $10985.5378(2)$ & $10962.7701(9)$ & $10917.4998(2)$ & $10897.5516(4)$ & $10993.8404(-4)$ \\
$5_{14}-4_{23}$ & $12787.4060(0)$ & $12745.3236(4)$ & $12712.9457(3)$ & $12723.5439(1)$ & $12792.7537(3)$ \\
$5_{15}-4_{04}$ & $10999.8348(2)$ & $10978.5394(-4)$ & $10931.2609(1)$ & $10908.1982(-2)$ & $11008.5606(-6)$ \\
\hline
\end{tabular}

Table 2. Rotational constants and quartic centrifugal distortion constants of the ${ }^{13} \mathrm{C}$-isotopomers of 1,2,3-trifluorobenzene with standard errors.

\begin{tabular}{cccccc}
\hline & ${ }^{13} \mathrm{C}_{2}$ & ${ }^{13} \mathrm{C}_{3}$ & ${ }^{13} \mathrm{C}_{4}$ & ${ }^{13} \mathrm{C}_{5}$ & ${ }^{12} \mathrm{C}$ \\
\hline${ }_{A} / \mathrm{MHz}$ & $2315.26797(10)$ & $2319.63903(10)$ & $2298.06711(11)$ & $2272.17274(13)$ & $2319.60029(6)$ \\
${ }_{B} / \mathrm{MHz}$ & $1757.93383(14)$ & $1749.22591(17)$ & $1748.64646(17)$ & $1757.84791(25)$ & $1757.83010(8)$ \\
$C / \mathrm{MHz}$ & $999.16620(9)$ & $997.15481(8)$ & $992.96022(9)$ & $991.02463(7)$ & $999.93756(10)$ \\
$\Delta_{J} / \mathrm{kHz}$ & $0.131(3)$ & $0.129(8)$ & $0.126(3)$ & $0.122(7)$ & $0.128(2)$ \\
$\Delta_{J K} / \mathrm{kHz}$ & $-0.190(10)$ & $-0.194(11)$ & $-0.174(10)$ & $-0.176(26)$ & $-0.177(8)$ \\
$\Delta_{K} / \mathrm{kHz}$ & $0.070(8)$ & $0.072(9)$ & $0.061(9)$ & $0.068(19)$ & $0.064(7)$ \\
$\delta_{J} / \mathrm{kHz}$ & $0.016(2)$ & $0.011(2)$ & $0.011(2)$ & $0.019(4)$ & $0.007(1)$ \\
$\delta_{K} / \mathrm{kHz}$ & $0.024(8)$ & $0.028(10)$ & $0.035(11)$ & $0.026(17)$ & $0.038(10)$ \\
\hline
\end{tabular}

axes system of the $i$-th isotopomer; $v_{1}, v_{2}, \ldots, v_{30}$ vibrational quantum numbers corresponding to the 30 normal vibrations of trifluorobenzene). The coordinates $a_{i, n}$ etc. are functions of the normal coordinates of the isotopomer under consideration. In principle the effective rotational constants may be expanded around their equilibrium values $A_{e}^{(i)}, B_{e}^{(i)}, C_{e}^{(i)}$ as power series in the vibrational quantum numbers (see [7], Chapt. XIII, 6):

$$
A_{v_{1}, \ldots v_{30}}^{(i)}=A_{e}^{(i)}-\sum_{s=1}^{30} \alpha_{i, s}^{a} \cdot\left(v_{s}+\frac{1}{2}\right)+\ldots \text { e.t.c. }
$$

If experimental values for the effective rotational constants were available also for molecules in all singly excited vibrational states, (3) could be solved for the equilibrium values $A_{e}^{(i)}, B_{e}^{(i)}, C_{e}^{(i)}$, and these in turn could be used in an iterative non linear least squares fit of the equilibrium internal coordinates. With only vibronic ground state rotational constants available at present, we had to use a different approach. We tried two types of fits of the structural parameters to the observed rotational constants. Both approaches had in common that the rotational constants were treated as if the molecule were rigid and planar (all $c_{i, n}$-coordinates were set to zero), i. e. we treated the rotational constants as if they were 
given by

$$
\begin{aligned}
A^{(i)} & =\frac{h}{8 \pi^{2}} \cdot \frac{1}{\sum m_{n} \cdot b_{i, n}^{2}}, \\
B^{(i)} & =\frac{h}{8 \pi^{2}} \cdot \frac{1}{\sum m_{n} \cdot a_{i, n}^{2}}, \\
C^{(i)} & =\frac{h}{8 \pi^{2}} \cdot \frac{1}{\sum m_{n} \cdot\left(a_{i, n}^{2}+b_{i, n}^{2}\right)} .
\end{aligned}
$$

In the first approach the internal coordinates were directly fitted to the rotational constants. This approach leads to a so called $r_{0}$-structure [7]. The second approach, first introduced by the Zürich group [18], involves fits to the differences between observed rotational constants. Specifically, in the second approach, the structural parameters were fitted to the rotational constants of the most abundant isotopomer (the parent molecule) and to the differences between the rotational constants of the ${ }^{13} \mathrm{C}$-isotopomers and the parent molecule, i. e. to

$$
\begin{aligned}
& \left.A_{(13} C_{(i)}\right)-A_{\text {parent }} \\
& B_{(13} C_{(i)}-B_{\text {parent }} \\
& C_{\left({ }^{13} C_{(i)}\right)}-C_{\text {parent }}(i=2 \text { to } 5)
\end{aligned}
$$

This second approach is assumed to eliminate part of the model errors which are introduced by the neglect of vibrational effects. The reasoning is as follows: Since in the fairly rigid molecule 1,2,3trifluorobenzene, vibrational averaging is probably very similar in the parent species and in the ${ }^{13} \mathrm{C}$ isotopomers, i. e. since $\alpha_{\text {parent,s }} \approx \alpha_{i, s}$ (compare (3)), the $\alpha$-corrections will partly cancel and the differences in the effective rotational constants will come closer to the differences in the equilibrium values. As a consequence, the second approach may be expected to lead to structural parameters which come closer to the equilibrium values.

\section{b) Results and Discussion}

The fits of the internal coordinates to the observed rotational constants were carried out with Volker Typkes program MWSTR1. For the underlying theory compare [18]. If $\mathrm{C}_{2 v}$-symmetry is assumed, the structure of 1,2,3-trifluorobenzene may be described by 11 internal coordinates. Because of correlations, only 7 internal coordinates could be fitted simultaneously, i. e. we had to impose 4 constraints. They were chosen as follows:
Table 3. Fit 1. Structural parameters of 1,2,3-trifluorobenzene derived by the fit to the rotational constants of the parent molecule within the rigid rotor model (partial $r_{0}$-structure).

\begin{tabular}{|c|c|c|}
\hline bondlengths / $\AA$ & Fit 1 & Fit 2 \\
\hline $\mathrm{C}(1 / 2)-\mathrm{C}(2 / 3)$ & 1.3895 & 1.3845 \\
\hline $\mathrm{C}(3 / 1)-\mathrm{C}(4 / 6)$ & 1.3832 & 1.3815 \\
\hline $\mathrm{C}(4 / 5)-\mathrm{C}(5 / 6)$ & 1.3988 & 1.3954 \\
\hline $\mathrm{C}(1 / 3)-\mathrm{F}(1 / 3)$ & $1.3398 * *$ & 1.3459 \\
\hline $\mathrm{C}(2)-\mathrm{F}(2)$ & $1.3348 * *$ & 1.3409 \\
\hline $\mathrm{C}(4 / 6)-\mathrm{H}(4 / 6)$ & $1.082 *$ & $1.082 *$ \\
\hline $\mathrm{C}(5)-\mathrm{H}(5)$ & $1.082 *$ & $1.082 *$ \\
\hline $\mathrm{C}(2)-\mathrm{C}(5)$ & 2.807 & 2.777 \\
\hline bondangles $/^{\circ}$ & Fit 1 & Fit 2 \\
\hline $\mathrm{C}_{(1)} \mathrm{C}_{(2)} \mathrm{C}_{(3)}$ & 118.752 & 118.738 \\
\hline $\mathrm{C}_{(2 / 2)} \mathrm{C}_{(3 / 1)} \mathrm{C}_{(4 / 6)}$ & 121.498 & 121.611 \\
\hline $\mathrm{C}_{(3 / 1)} \mathrm{C}_{(4 / 6)} \mathrm{C}_{(5 / 5)}$ & 118.680 & 118.687 \\
\hline $\mathrm{C}_{(4)} \mathrm{C}_{(5)} \mathrm{C}_{(6)}$ & 120.892 & 120.768 \\
\hline$F_{(1 / 3)} C_{(1 / 3)} C_{(2 / 2)}$ & 118.093 & 118.268 \\
\hline $\mathrm{F}_{(1 / 3)} \mathrm{C}_{(1 / 3)} \mathrm{C}_{(4 / 6)}$ & 120.409 & 120.121 \\
\hline $\mathrm{F}_{(2 / 2)} \mathrm{C}_{(2 / 2)} \mathrm{C}_{(1 / 3)}$ & 120.624 & 120.681 \\
\hline $\mathrm{H}_{(4 / 6)} \mathrm{C}_{(4 / 6)}^{-15 / 5)} \mathrm{C}_{(5 / 5)}$ & $121.96^{*}$ & $121.96 *$ \\
\hline $\mathrm{H}_{(4 / 6)} \mathrm{C}_{(4 / 6)} \mathrm{C}_{(1 / 3)}$ & 119.360 & 119.353 \\
\hline $\mathrm{H}_{(5 / 5)} \mathrm{C}_{(5 / 5)} \mathrm{C}_{(4 / 6)}$ & 119.554 & 119.616 \\
\hline \multicolumn{3}{|c|}{ 1. $r_{\mathrm{C}_{(44} \mathrm{H}_{(4)}}=1.082 \AA$} \\
\hline \multicolumn{3}{|c|}{ 2. $r_{\mathrm{C}_{(5,} \mathrm{H}_{(5)}}=1.082 \AA$} \\
\hline \multicolumn{3}{|c|}{ 3. $r_{\mathrm{C}_{(2)} \mathrm{F}_{(2)}}=r_{\mathrm{C}_{(1)} \mathrm{F}_{(1)}}-0.005 \AA$} \\
\hline \multicolumn{3}{|c|}{ 4. $\angle \mathrm{H}_{(4 / 6)} \mathrm{C}_{(4 / 6)} \mathrm{C}_{(5 / 5)}=121.96^{\circ}$} \\
\hline
\end{tabular}

Fit 2. Structural parameters derived by the fit to the rotational constants of the parent molecule and to the differences between the rotational constants of the parent molecule and the rotational constants of the ${ }^{13} \mathrm{C}$-isotopomers (hybrid between a partial $r_{0}$-structure and a $\mathrm{r}_{\mathrm{S}}$-structure).

The third constraint, i. e. the $0.005 \AA$ difference between the CF-bonds, was imposed in view of the earlier results obtained for related compounds by Jochims [14] and Stiefvater [19]. It is also in accordance with the results of an ab initio SCF/HF/6-311G equilibrium structure calculation with the GAUSSIAN 92 program package [20]. Such a calculation predicts a $0.005 \AA$ difference for the equilibrium values. The fourth constraint was imposed in view of Stiefvaters results obtained for 1,2-difluorobenzene and 1,3-difluorobenzene. We simply assumed that the $\mathrm{H}_{(4 / 6)} \mathrm{C}_{(4 / 6)} \mathrm{C}_{(5 / 5)}$ bond angle may be approximated as the average of the corresponding values observed in ortho- and meta-difluorobenzene.

In Table 3 and Fig. 4 we present the results of our fits. The differences between the values in the first 


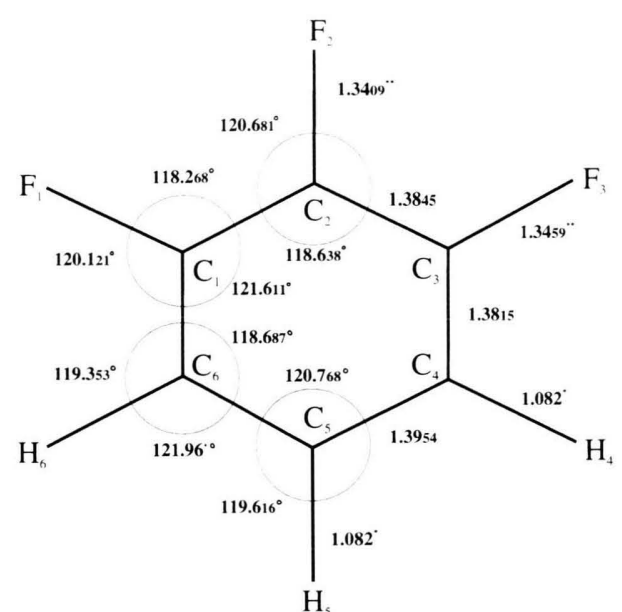

Fig. 4. Partial $r_{0}$-structure of 1,2,3-trifluorobenzene derived by the fit of 7 structural parameters to the rotational constants of the parent molecule and to the differences between the rotational constants of the parent molecule and the ${ }^{13} \mathrm{C}$-isotopomers $\left(*\right.$ assumed; $* * r_{\mathrm{C}(2)-\mathrm{F}(2)}=r_{\mathrm{C}(1 / 3)-\mathrm{F}(1 / 3)}$ $-0.005 \AA$ A).

column of Table 3 (fit to the rotational constants) and the corresponding values in the second column (fit to the rotational constants of the parent species and to the differences between the rotational constants of the ${ }^{13} \mathrm{C}$-species and the corresponding values of the parent species) illustrates trends in the effects of vibrational averaging: vibrational averaging tends to increase the effective bond distances between the carbon atoms of the ring but tends to decrease the effective CF-bond distances. The latter "bond-shrinkage" most likely reflects the effects of vibrational averaging over the out-of-plane bending vibrations.

The observed overall pattern of the benzene-ring distortions caused by the fluorine substituents looks familiar. As compared to benzene, the annular bond angles at the $\mathrm{C}_{(1)}$ and $\mathrm{C}_{(3)}$ positions are enlarged, while the $\left.\mathrm{C}_{(1)} \mathrm{C}_{(2)}\right)^{-}$and $\mathrm{C}_{(2)} \mathrm{C}_{(3)^{-}}$bonds are slightly shortened. Nygaard et al. [21, 22], in their pioneering work on fluorobenzene, have rationalized both distortions within a simple hybridization model. Within this model, fluorine substitution slightly changes the original local $\mathrm{sp}^{2}$ hybridization at the $\mathrm{C}$ atoms in the following way. The sp-hybrid pointing towards the electronegative $\mathrm{F}$ gains in $\mathrm{p}$-character while, as a consequence (orthogonality of the local sp-hybrid orbitals), the orbitals pointing towards the neighbouring $\mathrm{C}$ atoms gain in s-character and become more sp-like i. e. linear.

In a subsequent study of the deuterated 1,2,3trifluorobenzenes we hope to reduce the remaining ambiguities introduced by the constraints which had to be imposed during the fits of the structural parameters. To provide an impression of these ambiguities we give an example. If, for instance, one would assume the $\mathrm{CF}$ bond distances to be equal rather than to differ by $0.005 \AA$, Fit 2 would lead to

$$
\begin{aligned}
& r_{\mathrm{C}_{(1 / 2)} \mathrm{C}_{(2 / 3)}}=1.3830 \AA, \\
& r_{\mathrm{C}_{(1 / 3)} \mathrm{C}_{(6 / 4)}}=1.3844 \AA, \\
& r_{\mathrm{C}_{(4 / 5)} \mathrm{C}_{(5 / 6)}}=1.3954 \AA
\end{aligned}
$$

for the $\mathrm{CC}$ bond distances, and to

$$
\begin{aligned}
& \angle \mathrm{C}_{(1)} \mathrm{C}_{(2)} \mathrm{C}_{(3)}=118.850^{\circ}, \\
& \angle \mathrm{C}_{(2 / 3)} \mathrm{C}_{(3 / 1)} \mathrm{C}_{(4 / 6)}=121.500^{\circ}, \\
& \angle \mathrm{C}_{(3 / 1)} \mathrm{C}_{(4 / 6)} \mathrm{C}_{(5 / 5)}=118.692^{\circ}, \\
& \angle \mathrm{C}_{(4)} \mathrm{C}_{(5)} \mathrm{C}_{(6)}=120.767^{\circ}
\end{aligned}
$$

for the annular bond angles, i. e. the optimized annular bond angles would be changed by $0.1^{\circ}$ at most. In the study of the deuterated species we also intend to present the results of high level ab initio calculations which are carried out in parallel to the spectroscopical investigation. While the HF-calculation with the 6-311G - basis produced a planar equilibrium geometry, a higher level partial geometry optimization including electron correlation, in which the geometry of the aromatic ring was fixed to the values given in the second column of Table 3 , indicates that the equilibrium configuration of 1,2,3-trifluorobenzene might slightly deviate from planarity [23] .

\section{Acknowledgements}

The authors would like to thank Dr. R. Jaquet for many fruitful discussions concerning quantum chemical aspects and Prof. A. Guarnieri for critically reading the manuscript. Funds were provided by Deutsche Forschungsgemeinschaft, Fonds der Chemie, and Land Schleswig-Holstein. Also free computer time at the Computer Center of the University and the fruitful cooperation with its staff members are gratefully acknowledged. 
[1] D. Hübner, M. Stolze, and D. H. Sutter, Z. Naturforsch. 36a, 332 (1981).

[2] W. H. Stolze, M. Stolze, D. Hübner, and D. H. Sutter, Z. Naturforsch. 37a, 1165 (1982).

[3] O. Böttcher and D. H. Sutter, Z. Naturforsch. 43a, 47 (1988).

[4] W. H. Stolze and D. H. Sutter, Z. Naturforsch. 44a, 687 (1989).

[5] D. H. Sutter and W. H. Flygare, The Molecular Zeeman Effect, in "Topics in Current Chemistry", Vol. 63, 89 - 196 (1976).

[6] M. Onda, H. Mukaida, H. Akiba, M. Mori, H. Miyazaki, and I. Yamaguchi, J. Mol. Spectrosc. 169, 480 (1995).

[7] Walter Gordy and Robert L. Cook, Microwave Molecular Spectra, $3^{\text {rd }}$ Edition, John Wiley and Sons, New York 1984, Chapt. XIII.

[8] U. Andresen, H. Dreizler, U. Kretschmer, W. Stahl, and C. Thomsen, Fresenius' J. Anal. Chem. 347, 272 (1994).

[9] Jens Uwe Grabow, Diploma - Thesis, University of Kiel (1989).

[10] U. Kretschmer, D. Consalvo, A. Knaack, W. Schade, W. Stahl, and H. Dreizler, Manuscript submitted to J. Mol. Phys.

[11] N. Hansen, Diploma - Thesis, University of Kiel (1995).

[12] J. U. Grabow and W. Stahl, Z. Naturforsch. 45a, 1043 (1990).
[13] T. J. Balle and W. H. Flygare, Rev. Sci. Instrum. 52, 33 (1981).

[14] Ekkehard Jochims, Diploma - Thesis, University of Kiel (1993).

[15] E. Jochims, J.-U. Grabow, and W. Stahl, J. Mol. Spectrosc. 158, 278 (1992).

[16] H. Johns and V. Typke, Z. Naturforsch. 36a, 1057 (1981).

[17] J. K. G. Watson, Aspects of Quartic and Sextic Centrifugal Effects on Rotational Energy Levels, in "Vibrational Spectra and Structure " (J. R. Durig editor), Vol. 6, Chapt. 1, p. 2 - 89, Elsevier, Amsterdam 1977.

[18] P. Nösberger, A. Bauder, and Hs. H. Günthard, Chem. Phys. 1, 418 (1973).

[19] O. L. Stiefvater, Z. Naturforsch. 43a, 147-154 and 155-163 (1988).

[20] GAUSSIAN 92, M.J. Frisch, M. Head-Gordon, G. W. Trucks, J. B. Foresman, H. B. Schlegel, M. A. Robb, J. S. Binkley, C. Gonzales, D. J. Defrees, D. J. Fox, R. A. Whiteside, R. Seeger, C. F. Melius, J. Baker, R. L. Martin, L. R. Kahn, J. J. P. Stewart, S. Topiol, and J. A. Pople, Gaussian Inc., Pittsburgh PA 1992.

[21] L. Nygaard, E. R. Hansen, J. Rastrup-Andersen, and J. O. Sorensen, Spectrochim. Acta 23A, 2813 (1967).

[22] L. Nygaard, I. Bojesen, T. Pedersen, and J. RastrupAndersen, J. Mol. Struct. 2, 209 (1968).

[23] Ralph Jaquet, University of Siegen, private communication. 